Article

\title{
Improved Decision Fusion Model for Wireless Sensor Networks over Rayleigh Fading Channels
}

\author{
Ali Jamoos \\ Department of Electronic and Communication Engineering, Al-Quds University, P.O. Box 20002, Jerusalem, \\ Palestine; ali.jamoos@staff.alquds.edu or ali.jamoos@ieee.org; Tel.: +970-597725063 \\ Academic Editor: Manoj Gupta \\ Received: 22 January 2017; Accepted: 8 March 2017; Published: 10 March 2017
}

\begin{abstract}
This paper deals with decision fusion in wireless sensor networks (WSNs) over Rayleigh fading channels. The likelihood ratio test (LRT) is considered as the optimal fusion rule when applied at the fusion center (FC). However, applying the LRT at the FC requires both the channel state information (CSI) and the local sensors' performance indices. Acquiring such information is considered as an overhead in energy and bandwidth constrained systems such as WSNs. To avoid these drawbacks, we propose a modification to the traditional three-layer system model of a WSN where the LRT is applied as a local decision making method at the sensors level. Applying the LRT at the sensors level does not require the CSI or the local sensors' performance indices. It only requires the signal-to-noise ratio (SNR). Moreover, a new fusion rule based on selection combining (SC) is suggested. This fusion method has the lowest complexity when compared to other diversity combining based fusion rules such as the equal gain combiner (EGC) and the maximum ratio combiner (MRC). Simulation results show that the performance of the proposed model outperforms the traditional model. In addition, applying the EGC at the FC in the proposed model provides comparable performance to the traditional model that applies the LRT at the FC.
\end{abstract}

Keywords: wireless sensor networks (WSNs); decisions fusion; fading channels; likelihood ratio test (LRT); EGC; MRC; SC

\section{Introduction}

Pervasive sensing technology has the potential to enhance information gathering and processing in diverse applications. A typical wireless sensor network (WSN) employs multiple sensors, each equipped with devices capable of sensing, processing, and communication [1-4]. The advantages of WSNs include flexibility in deployment and scalability, low cost and fast initial set-up. Recent advances in micro-sensors have enabled WSN to a wide range of applications, such as battlefield surveillance, environmental monitoring, and health care applications [5-8]. Significant challenges exist and need to be addressed in order for the envisaged application to become a reality. For instance, the individual sensors are incredibly resource constrained. They have limited storage capacity and communication bandwidth. In addition, in many WSN applications, sensors operate on irreplaceable power supply, making it necessary to conserve power for prolonged lifetime. From an energy-consumption perspective, transmitting or receiving one kilobyte of information is equivalent to computing 3 million of instructions [9], so it is recommended to make a computation at the sensor level instead of transmitting whenever it is possible.

Decisions fusion represents a formal framework that deals with data collected from different resources to obtain a greater quality of global decision about a certain phenomena. Decisions fusion with uncertainty has been investigated and a Bayesian sampling approach has been proposed to address this issue [10]. In [11,12], the optimum fusion rule has been obtained under the conditional independence assumption. A fusion of decisions that are correlated to each other has been studied 
in [13]. Distributed detection in a constrained system has been considered in [14]. Decisions fusion in WSN operated with a multiple input multiple output (MIMO) channel has been investigated in $[15,16]$. Universal decentralized detection in a bandwidth-constrained sensor network with binary decisions made by sensor nodes has been constructed in [17]. Universal detectors for the decision fusion problem have also been considered in [18].

The distributed detection problem in WSNs has been studied using two kinds of communication channels: the traditional parallel access channel (PAC) in which each sensor has a dedicated independent channel to fusion center (FC) and the multiple access channel (MAC) in which the FC receives a coherent superposition of the sensor transmissions $[19,20]$. On the one hand, for networks with a large number of sensors, PAC implies a large bandwidth requirement and a large detection delay. On the other hand, the bandwidth requirement of MAC does not depend on the number of sensors, but due to the additive nature of the channel, the received signal at the FC is usually not sufficient for reliable detection.

Channel-aware distributed detection has been proposed in [21,22], which integrates the wireless channel conditions in algorithm design. Channel-aware distributed detection has also been considered for a decode-then-fuse model and parallel access channels in [23], as well as for a MIMO channel model with both instantaneous and statistical channel state information (CSI) in [24-26]. The fusion of decisions transmitted over Rician fading channels has been investigated in [27]. In [28], five different fusion rules have been investigated in a three-layer parallel access WSN fusion model over Rayleigh fading channels. These fusion rules are the likelihood ratio test (LRT), equal gain combiner (EGC), maximum ratio combiner (MRC), Chair-Varshney and the likelihood ratio test based on channel statistics (LRT-CS). It is shown in [28] that the LRT fusion rule is the optimum fusion rule. Nevertheless, the LRT requires the CSI which should be estimated. In addition, the local sensors' performance indices must be transmitted by each sensor to the FC. Thus, applying LRT at the FC is too costly since WSNs are known to be a constrained system in terms of communication bandwidth and energy consumption. On the other hand, the EGC is a simple fusion rule since it does not require any knowledge about the channel or the local sensors' performance indices. Optimal local sensor detection does not necessarily yield a global optimal detection and compromises should be made with each other as well as the fusion rule at the FC.

Taking into account the limitation on energy and bandwidth of WSNs, we propose in this paper a modification to the existing three-layer PAC decision fusion model for WSNs where the LRT is applied locally to each sensor. Applying the LRT at the sensors level does not require the CSI or transmitting local sensors' performance indices from each sensor to the FC. It only requires the instantaneous channel signal-to-noise ratio (SNR). Moreover, we propose to apply selection combining (SC) as decision fusion method at the FC.

The remainder of the paper is organized as follows. Section 2 presents the existing three-layer WSN system model and the state-of-the-art decision fusion rules. Section 3 introduces the proposed WSN system model. In Section 4, a comparative simulation study is carried out between the proposed WSN model and the existing WSN model. Conclusions are drawn in Section 5.

\section{Traditional Three-Layer WSN System Model}

The traditional three-layer PAC decision fusion model for WSNs in the presence of additive white Gaussian noise (AWGN) and fading channels is illustrated in Figure 1. There are two hypotheses under testing, $H_{1}$ (target present), and $H_{0}$ (target absent). Each sensor receives noisy measurements and processes these measurements in order to make decisions regarding the hypothesis under testing. Then, each sensor transmits the obtained binary decision to the FC through parallel access channels which undergo AWGN and Rayleigh fading.

For WSNs with limited resources, the effect due to channel fading and noise renders the information received at the FC not completely reliable. Corruption on the received local decisions will 
lead to performance loss if they are not properly considered. The traditional three-layer parallel WSN system model can be described in detail as follows.

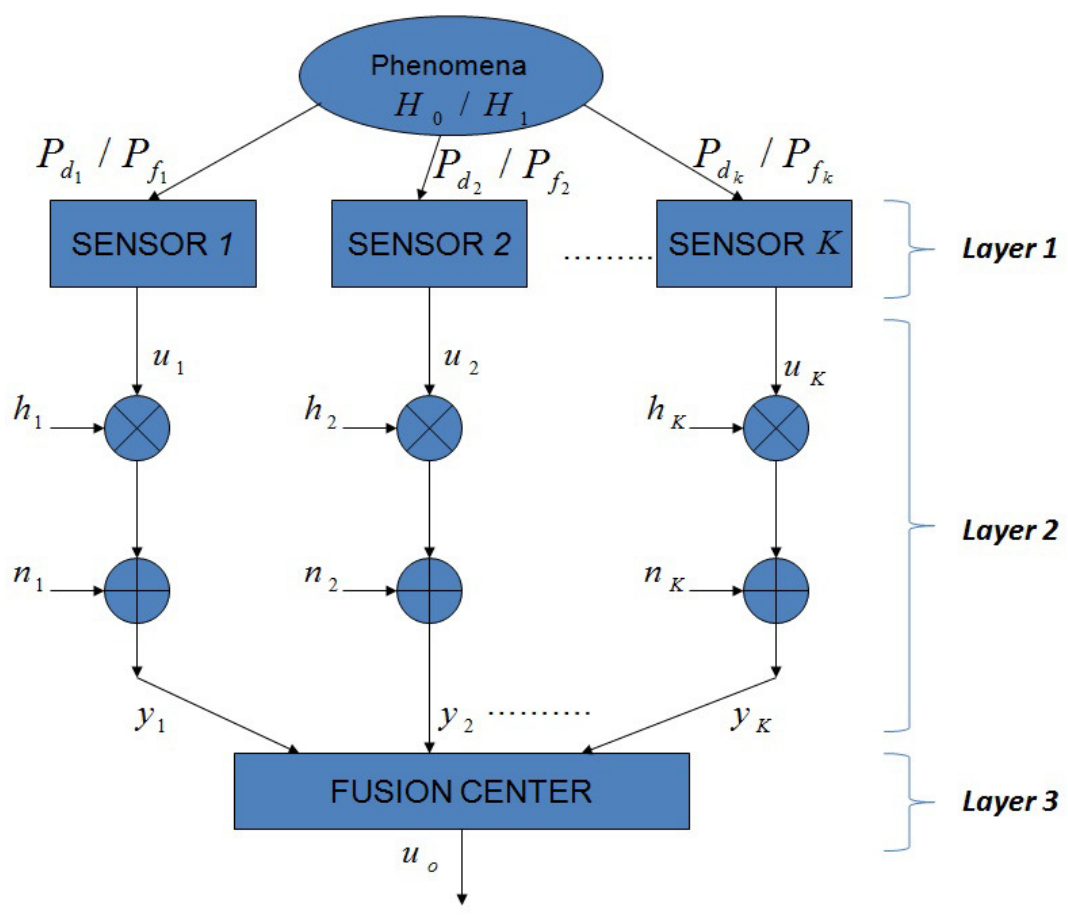

Figure 1. Traditional three-layer parallel WSN system model [28].

\subsection{Layer 1: Sensors}

In this layer, all of the local sensors receive noisy measurements regarding a specific hypothesis. In this work, we assume that the observations are conditionally independent of each other. After receiving its observation, $x_{k}$, each sensor, $k$, makes a hard (binary) decision: $u_{k}=1$ is sent if $H_{1}$ is decided, and $u_{k}=-1$ is sent otherwise, where $k=1, \ldots, K$ and $K$ is the total number of sensors in the network. The hard binary decision can be formalized as follows:

$$
u_{k}= \begin{cases}1 & : x_{k}>0 \\ -1 & : x_{k}<0\end{cases}
$$

In addition, we assume that each sensor makes a binary decision based on its own observation. The detection performance of each local sensor node can be characterized by its corresponding probability of detection and probability of false alarm, denoted by $P_{d_{k}}$ and $P_{f_{k}}$, respectively, for the $k$ th sensor:

$$
\begin{aligned}
& P_{d_{k}}=P\left(u_{k}=1 \mid H_{1}\right), \\
& P_{f_{k}}=P\left(u_{k}=1 \mid H_{0}\right) .
\end{aligned}
$$

In general, these pairs $\left(P_{d_{k}}, P_{f_{k}}\right)$ may not be identical and they are functions of SNRs as well as the detection threshold at each sensor.

\subsection{Layer 2: Fading and Noisy Channels}

Decisions at local sensors, denoted by $u_{k}$ for $k=1, \ldots, K$, are transmitted over parallel channels that are assumed to undergo independent fading. Since most of the WSNs operate at short range and low bit rate due to power and energy limitations, the fading channels are assumed to be flat. We further assume phase coherent reception, thus the effect of a fading channel is further simplified as 
a real scalar multiplication given that the transmitted signal is assumed to be binary. The statistics of the real scalar, denoted by $h_{k}$, is determined by the fading type. For example, for homogeneous scattering background, Rayleigh distribution best describes the envelope of a fading signal. In the development of fusion rules, the gain of the fading channel is considered as an unknown constant during the transmission of a single local decision. We assume that the channel noise is AWGN and uncorrelated from channel to channel. Thus, the input to the FC for the $k$ th sensor is given by

$$
y_{k}=h_{k} u_{k}+n_{k}
$$

where $h_{k}$ is Rayleigh distributed fading channel gain with unit power (i.e., $E\left[h_{k}^{2}\right]=1$ ) and $n_{k}$ is a zero-mean Gaussian random variable with variance $\sigma^{2}$.

\subsection{Layer 3: Fusion Center}

The FC is the most important part in the WSN system which makes a global decision $u_{o}$ regarding a certain phenomena based on the received $y_{k}$ data for all $k$. This is done by making use of a certain fusion rule applied at the FC. According to the used fusion rule at the FC, some other parameters may be required in order to make the global decision such as the CSI and the local sensors' performance indices. The function of the FC after forming a certain statistic $\Lambda$ can be expressed as:

$$
u_{o}= \begin{cases}1 & : \Lambda>T, \\ -1 & : \Lambda<T,\end{cases}
$$

where $T$ is the decision threshold at the FC.

To facilitate our comparisons later, we give a brief review here of the fusion rules developed in $[22,28]$. Namely, the likelihood ratio test (LRT), equal gain combiner (EGC), maximum ratio combiner (MRC), Chair-Varshney and the likelihood ratio test based on channel statistics (LRT-CS). In addition, the so-called selection combining will also be investigated as a fusion rule.

\subsubsection{The Optimal LRT}

By assuming instantaneous channel state knowledge regarding the fading channel and the local sensor performance indices, i.e., the $P_{d_{k}}$ and $P_{f_{k}}$ values, the optimal LRT-based fusion rule has been derived in [22], with the fusion statistic $\Lambda$ given by

$$
\begin{aligned}
\Lambda(\mathbf{y}) & =\log \left[\frac{f\left(\mathbf{y} \mid H_{1}\right)}{f\left(\mathbf{y} \mid H_{0}\right)}\right] \\
& =\sum_{k=1}^{K} \log \left[\frac{P_{d_{k}} e^{-\frac{\left(y_{k}-h_{k}\right)^{2}}{2 \sigma^{2}}}+\left(1-P_{d_{k}}\right) e^{-\frac{\left(y_{k}+h_{k}\right)^{2}}{2 \sigma^{2}}}}{P_{f_{k}} e^{-\frac{\left(y_{k}-h_{k}\right)^{2}}{2 \sigma^{2}}}+\left(1-P_{f_{k}}\right) e^{-\frac{\left(y_{k}+h_{k}\right)^{2}}{2 \sigma^{2}}}}\right] .
\end{aligned}
$$

The LRT value is then compared with a threshold at the FC to make a final decision. While the form of the LRT based fusion statistic is straightforward to implement, it does need both the local sensor performance indices and complete channel knowledge.

\subsubsection{Chair-Varshney Fusion Rule}

In [28], the following statistic, termed as the Chair-Varshney fusion statistic, has been shown to be a high-SNR approximation to $\Lambda$

$$
\Lambda_{1}=\sum_{\operatorname{sign}\left(y_{k}\right)=1} \log \frac{P_{d_{k}}}{P_{f_{k}}}+\sum_{\operatorname{sign}\left(y_{k}\right)=-1} \log \frac{1-P_{d_{k}}}{1-P_{f_{k}}} .
$$


$\Lambda_{1}$ does not require any knowledge regarding the channel gain but does require $P_{d_{k}}$ and $P_{f_{k}}$ for all $k$. This approach, however, suffers significant performance loss at low channel SNR.

\subsubsection{MRC Fusion Rule}

It has been shown in [28] that, for small values of channel SNR, $\Lambda$ in Equation (5) reduces to

$$
\hat{\Lambda}_{2}=\sum_{k=1}^{K}\left(P_{d_{k}}-P_{f_{k}}\right) h_{k} y_{k}
$$

Furthermore, if the local sensors are identical, i.e., $P_{d_{k}}=P_{d}$ and $P_{f_{k}}=P_{f}$ for all $k \mathrm{~s}$, then $\Lambda$ further reduces to a form analogous to a MRC statistic

$$
\Lambda_{2}=\frac{1}{K} \sum_{k=1}^{K} h_{k} y_{k}
$$

$\Lambda_{2}$ in Equation (8) does not require the knowledge of $P_{d}$ and $P_{f}$ provide $P_{d}-P_{f}>0$. Knowledge of the channel gain is, however, required.

\subsubsection{EGC Fusion Rule}

Motivated by the fact that resembles a MRC statistic for diversity combining, a third alternative in the form of an EGC has been proposed in [28], which requires a minimum amount of information:

$$
\Lambda_{3}=\frac{1}{K} \sum_{k=1}^{K} y_{k}
$$

Interestingly enough, this simple alternative fusion rule outperforms both MRC and Chair-Varshney fusion rules for a wide range of SNR in terms of its detection performance [28].

\subsubsection{LRT-CS Fusion Rule}

A fusion rule based on the optimal LRT has been proposed in [28]. This fusion rule requires the knowledge about the wireless channel statistical characteristics instead of the instantaneous CSI. This fusion rule is given by

$$
\Lambda_{4}=\sum_{k=1}^{K} \log \left\{\frac{1+\left[P_{d_{k}}-Q\left(a y_{k}\right)\right] \sqrt{2 \pi} a y_{k} e^{\frac{\left(a y_{k}\right)^{2}}{2}}}{1+\left[P_{f_{k}}-Q\left(a y_{k}\right)\right] \sqrt{2 \pi} a y_{k} e^{\frac{\left(a y_{k}\right)^{2}}{2}}}\right\}
$$

where $a=1 /\left(\sigma \sqrt{1+2 \sigma^{2}}\right)$ and $Q(\cdot)$ is the complementary distribution function of the standard Gaussian.

The above fusion rule outperforms both the EGC and Chair-Varshney fusion rules and has better performance than the MRC fusion rule for most practical SNR values (except for very low SNR values) [28].

\subsubsection{SC Fusion Rule}

The selection combining (SC) fusion rule has lower implementation complexity compared to the MRC and the EGC, and it is based on selecting the branch that has the maximun instantaneous channel SNR as follows:

$$
\Lambda(\mathbf{y})=\max \left\{\left|y_{1}\right| \ldots\left|y_{K}\right|\right\}
$$




\section{Proposed Three-Layer WSN System Model}

In this section, we propose a modification to the traditional three-layer WSN system model by applying the optimal LRT as a local decision making method at the sensors level as shown in Figure 2 . Applying the LRT at the sensors level does not require the CSI or transmitting the local sensors performance indices, i.e., $P_{d_{k}}=P_{d}$ and $P_{f_{k}}=P_{f}$ for all $k$ s. Indeed, from the energy consumption perspective, transmitting or receiving one kilobyte of information is equivalent to computing 3 million instructions [9], so it is recommended to make a computation in the sensor level instead of transmitting whenever it is possible.

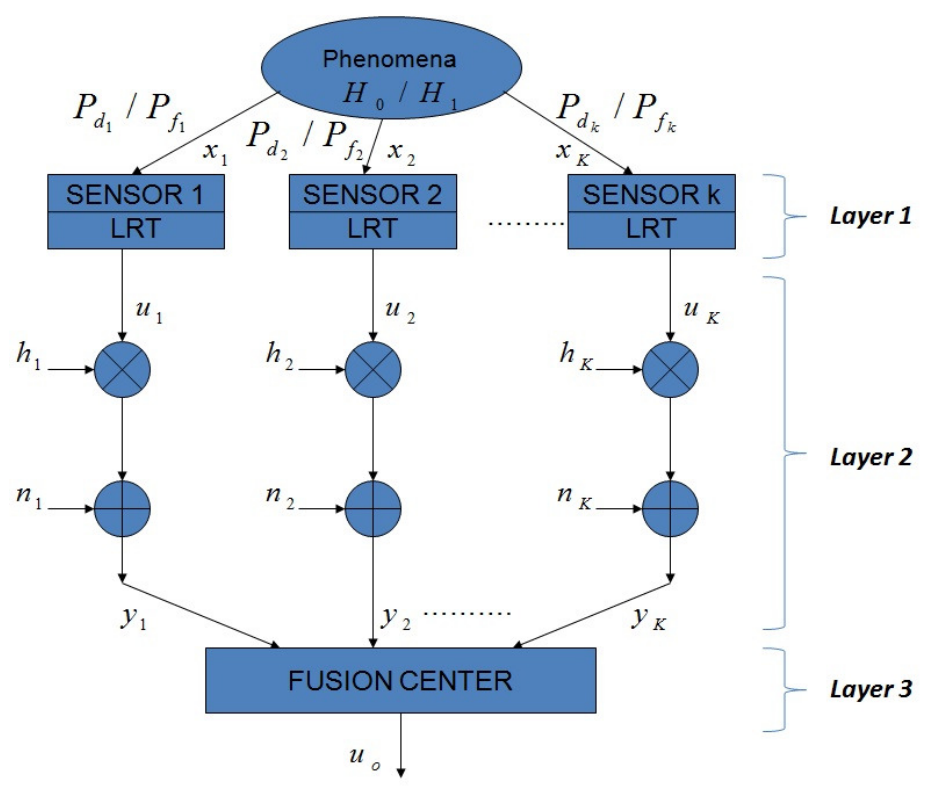

Figure 2. Proposed three-layer parallel WSN system model where the LRT is applied at the sensors level.

Assuming that there are two hypothesis under test $\left(H_{0}\right.$ and $\left.H_{1}\right)$, the measured noisy signal at each sensor can be described by the following equation:

$$
x_{k}= \begin{cases}s_{k}+w_{k} & : H_{1} \\ w_{k} & : H_{0}\end{cases}
$$

where $s_{k}$ represents the $k^{\text {th }}$ signal in the case of $H_{1}$ and $w_{k}$ is the corresponding sensor measurement additive white noise which is not necessarily Gaussian and whose variance is $\sigma_{w}^{2}$. Note that the sensor measurement noise $w_{k}$ is different from the channel noise $n_{k}$. In this work, we assume that the absence or the presence of the signal under interest $s_{k}$, i.e., $H_{1}$ or $H_{0}$, respectively, is described by either 1 or 0 as follows:

$$
s_{k}= \begin{cases}1 & : H_{1} \\ 0 & : H_{0}\end{cases}
$$

Then, the LRT based decision for each sensor can be written as

$$
\Lambda\left(x_{k}\right)=\log \frac{p\left(x_{k} \mid H_{1}\right)}{p\left(x_{k} \mid H_{0}\right)} \underset{H_{0}}{\stackrel{H_{1}}{\lessgtr}} T,
$$

where $T$ is the decision threshold. In the special case when the sensor measurement noise is assumed to be Gaussian, the measured information $x_{k}$ follows the normal distribution with zero-mean and variance of $\sigma_{w}^{2}$ in the case of $H_{0}$, and unit-mean with variance of $\sigma_{w}^{2}$ in the case $H_{1}$ as described by 


$$
\begin{array}{ll}
H_{0}: & x_{k} \sim N\left(0, \sigma_{w}^{2}\right), \\
H_{1}: & x_{k} \sim N\left(1, \sigma_{w}^{2}\right) .
\end{array}
$$

Assuming independent identically distributed (i.i.d) measurements among the sensors, the required probability density functions (PDFs) can be expressed as

$$
\begin{aligned}
& p\left(x_{k} \mid H_{0}\right)=\frac{1}{\sqrt{2 \pi \sigma_{w}^{2}}} \exp \left\{-\frac{1}{2}\left(\frac{x_{k}}{\sigma_{w}}\right)^{2}\right\}, \\
& p\left(x_{k} \mid H_{1}\right)=\frac{1}{\sqrt{2 \pi \sigma_{w}^{2}}} \exp \left\{-\frac{1}{2}\left(\frac{x_{k}-1}{\sigma_{w}}\right)^{2}\right\} .
\end{aligned}
$$

Substituting Equation (16) into Equation (14) yields to the LRT statistics $\Lambda\left(x_{k}\right)$ at sensor level

$$
\Lambda\left(x_{k}\right)=\log \left\{\frac{e^{-\frac{\left(x_{k}-1\right)^{2}}{2 \sigma_{w}^{2}}}}{e^{-\frac{\left(x_{k}\right)^{2}}{2 \sigma_{w}^{2}}}}\right\} .
$$

It can be noticed from Equation (17) that applying the LRT at the sensors level requires no prior information regarding the channel and no information about local sensors performance indices. It only requires the instantaneous channel SNR.

Now, each sensor makes a local decision regarding the absence or the presence of a certain hypothesis, $H_{1}$ and $H_{0}$, respectively, according to

$$
u_{k}= \begin{cases}1 & : \Lambda\left(x_{k}\right)>T \\ -1 & : \Lambda\left(x_{k}\right)<T\end{cases}
$$

It should be noted that, due to the sensor measurement noise Gaussian assumption, the traditional model in Figure 1 and the proposed model in Figure 2 are statistically equivalent if a varying threshold is considered at Equation (1). Nevertheless, in the following, the proposed model leads to improved performance by introducing an optimized local sensor threshold.

The performance of the LRT is mainly characterized by the probability of correctly recognizing the presence of the signal while it is actually present (probability of detection) and the probability of wrongly recognizing the signal as present while it is actually absent (probability of false alarm). The probability of a false alarm is defined as

$$
\begin{aligned}
P_{f} & =\int_{T}^{+\infty} p_{\Lambda}\left(\Lambda \mid H_{0}\right) d \Lambda \\
& =\int_{T}^{+\infty} \frac{1}{\sqrt{2 \pi \sigma_{w}^{2}}} e^{\frac{-\Lambda^{2}}{2 \sigma_{w}^{2}}} d \Lambda .
\end{aligned}
$$

Equation (19) is the integral of a Gaussian PDF, so it can be solved by using the definition of the error function $(\operatorname{erf}(x))$ [29], as follows:

$$
\operatorname{erf}(x)=\frac{2}{\sqrt{\pi}} \int_{0}^{x} e^{-t^{2}} d t
$$

changing the variables $t=\Lambda / \sqrt{2 \sigma_{w}^{2}}$, Equation (19) can be rewritten as

$$
\begin{aligned}
P_{f} & =\frac{1}{\sqrt{\pi}} \int_{T / \sqrt{2 \sigma_{w}^{2}}}^{+\infty} e^{-t^{2}} d t \\
& =\frac{1}{2}\left\{1-\operatorname{erf}\left(\frac{T}{\sqrt{2 \sigma_{w}^{2}}}\right)\right\} .
\end{aligned}
$$


Finally, Equation (21) can be solved to obtain the threshold $T$ in term of the inverse error function $\left(e r f^{-1}\right)$ as follows:

$$
T=\sqrt{2 \sigma_{w}^{2}} e r f^{-1}\left(1-2 P_{f}\right) .
$$

The probability of detection for the LRT is defined as follows:

$$
\begin{aligned}
P_{d} & =\int_{T}^{+\infty} p_{\Lambda}\left(\Lambda \mid H_{1}\right) d \Lambda \\
& =\int_{T}^{+\infty} \frac{1}{\sqrt{2 \pi \sigma_{w}^{2}}} e^{\frac{-(\Lambda-1)^{2}}{2 \sigma_{w}^{2}}} d \Lambda .
\end{aligned}
$$

Again, applying the definition of the error function in Equation (20) leads to

$$
P_{d}=\frac{1}{2}\left\{1-\operatorname{erf}\left(\frac{T-1}{\sqrt{2 \sigma_{w}^{2}}}\right)\right\} .
$$

Substituting Equation (22) in Equation (24) and make use of the complementary error function $(\operatorname{erf} c(x)=1-\operatorname{erf}(x))$ in order to eliminate the threshold $T$ leads to

$$
P_{d}=\frac{1}{2} \operatorname{erfc}\left\{\operatorname{erfc}^{-1}\left(2 P_{f}\right)-\sqrt{\frac{S N R}{2}}\right\} .
$$

Equation (25) describes the performance of the LRT in terms of fixed channel signal-to-noise ratio $S N R=1 / \sigma_{w}^{2}$ and fixed probability of false alarm $P_{f}$.

\section{Simulation Results}

In this section, the relative performance of different fusion rules applied at the FC in the proposed WSN system model is examined. In addition, a performance comparison between the traditional and the proposed WSN system model is carried out through simulation in order to obtain the receiver operating characteristics (ROC) curves for different fusion rules. Moreover, we studied the effect of various factors that may affect the performance of the FC such as the communication channel signal-to-noise ratio (SNR), total number of sensors in the network (i.e., $K$ ) and the local sensors' performance indices (i.e., $P_{d_{k}}$ and $P_{f_{k}}$ ).

In the first simulation scenario, ROC performance comparison among the various fusion rules applied at the proposed WSN system model is carried out. We assume that all sensors receive noisy measurements and all have the same SNR and thus having the same performance indices. Moreover, the channels between the sensors and the FC all have the same SNR. ROC curves for different fusion rules applied at the proposed WSN system model and channel SNR of $5 \mathrm{~dB}$ are plotted in Figure 3 . The local sensors' performance indices $P_{d_{k}}$ and $P_{f_{k}}$ are assumed to be 0.5 and 0.05 , respectively. The total number of sensors in the network is fixed at $K=8$. According to Figure 3, the LRT and the LRT-CS fusion rules have the best performance, whereas the SC fusion rule has the worst performance.

In the second simulation scenario, a comparison is made between the traditional and the proposed WSN model with the various fusion rules for variable channel SNR. We assume that the local sensors performance indices are identical and also the channels' SNR between the sensors and the FC are identical. From Figure 4, one can notice that the performance of the proposed model is better than that of the traditional model for the various fusion rules and for a wide range of channel SNR. While the LRT shows the best performance among the other fusion rules in both models, the EGC fusion rule applied at the proposed model provides a performance very similar to that of LRT-CS for a wide range of SNRs and better performance than other fusion rules such as MRC and Chair-Varshney fusion rules. Thus, applying the LRT at the sensors level and EGC at the FC can significantly raise the performance of the proposed model when compared to the traditional model. Moreover, Figure 4 shows that the proposed model could significantly increase the performance of MRC and SC fusion rules for high channel SNR. 


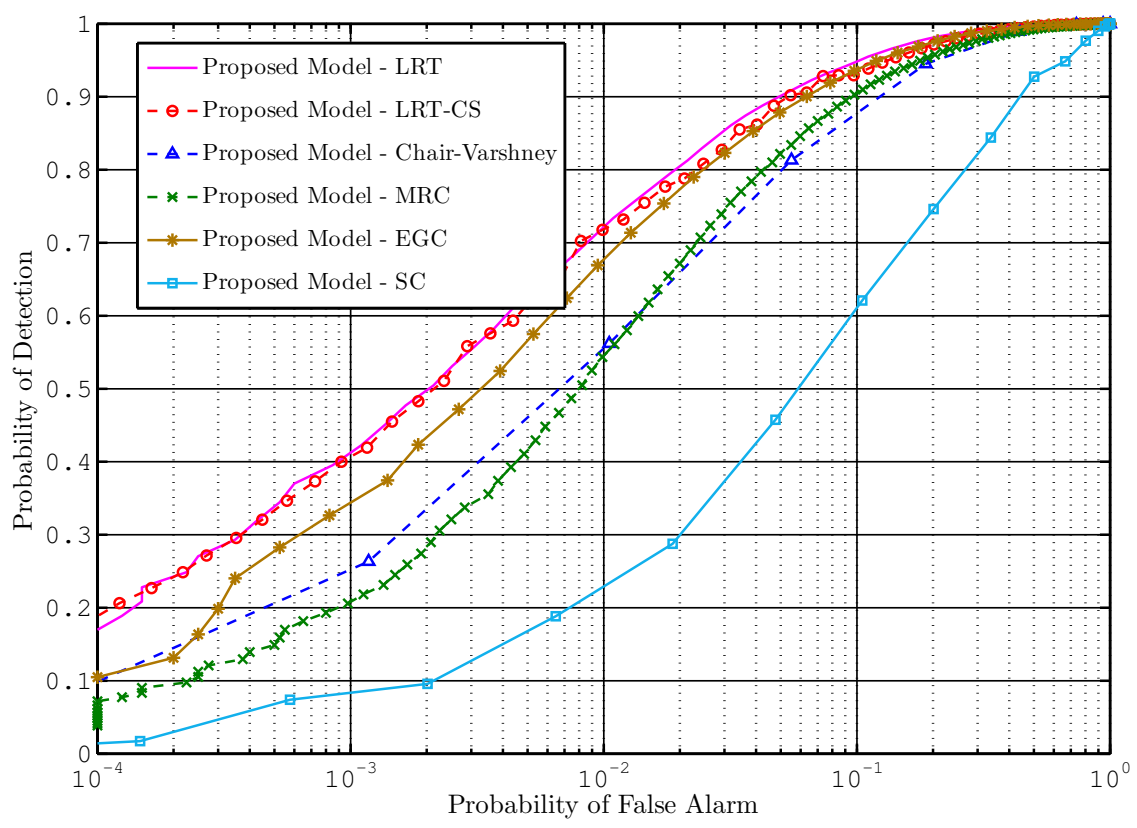

Figure 3. ROC curves for different fusion rules applied at the proposed WSN system model, average channel SNR $=5 \mathrm{~dB}, P_{d}=0.5, P_{f}=0.05$ and total number of sensors $K=8$.

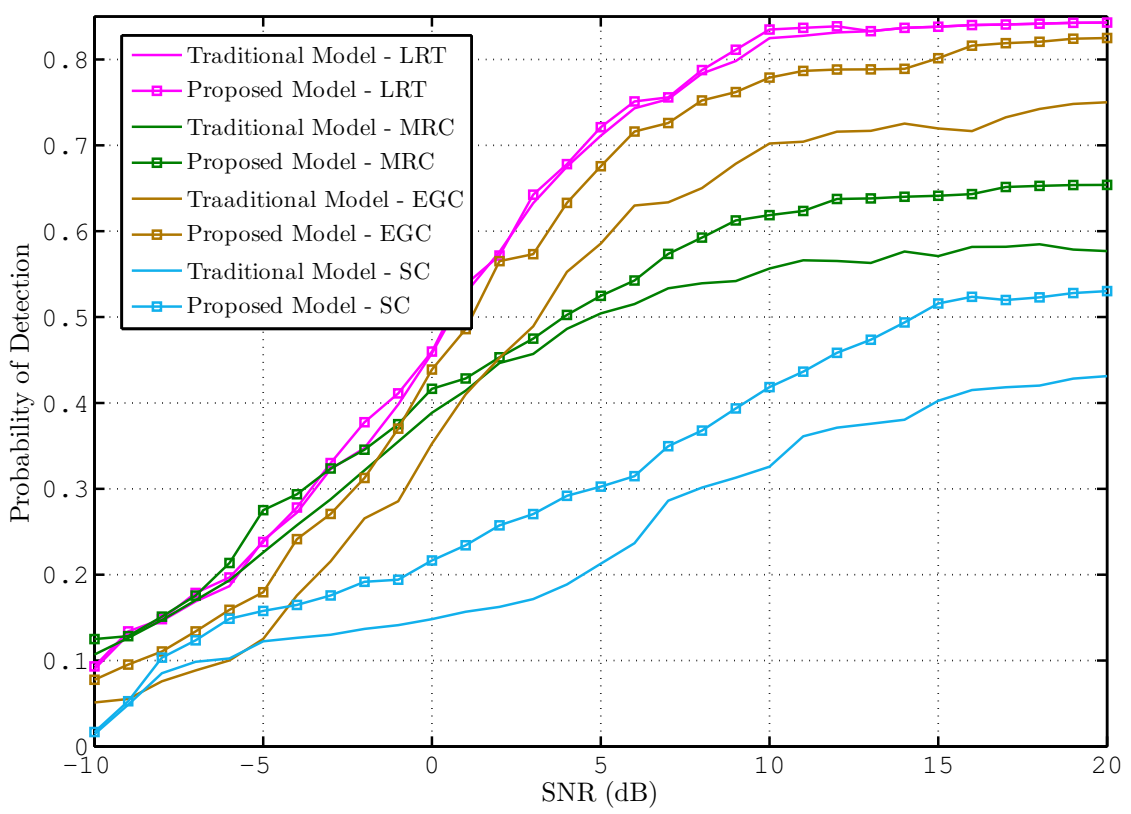

Figure 4. Probability of detection as a function of average channel SNR, $P_{d}=0.5, P_{f}=0.05$, and total number of sensors $K=8$.

In the third simulation experiment, we investigate the effect of number of sensors $K$ on the traditional and proposed model for the various fusion rules. We assume that the average channel SNR is fixed to $5 \mathrm{~dB}$, the local sensors have performance indices of $P_{d}=0.5, P_{f}=0.05$, and these indices are identical among all sensors. It can be observed from Figure 5 that the proposed model outperforms the traditional model for the various fusion rules and for different number of sensors $K=8$. In addition, one can notice that, even for small number of sensors $K$, the performance of the EGC applied at the proposed model is nearly the same to that of the optimum LRT and outperforms all other fusion rules and shows more robustness regarding the total number of sensors. 


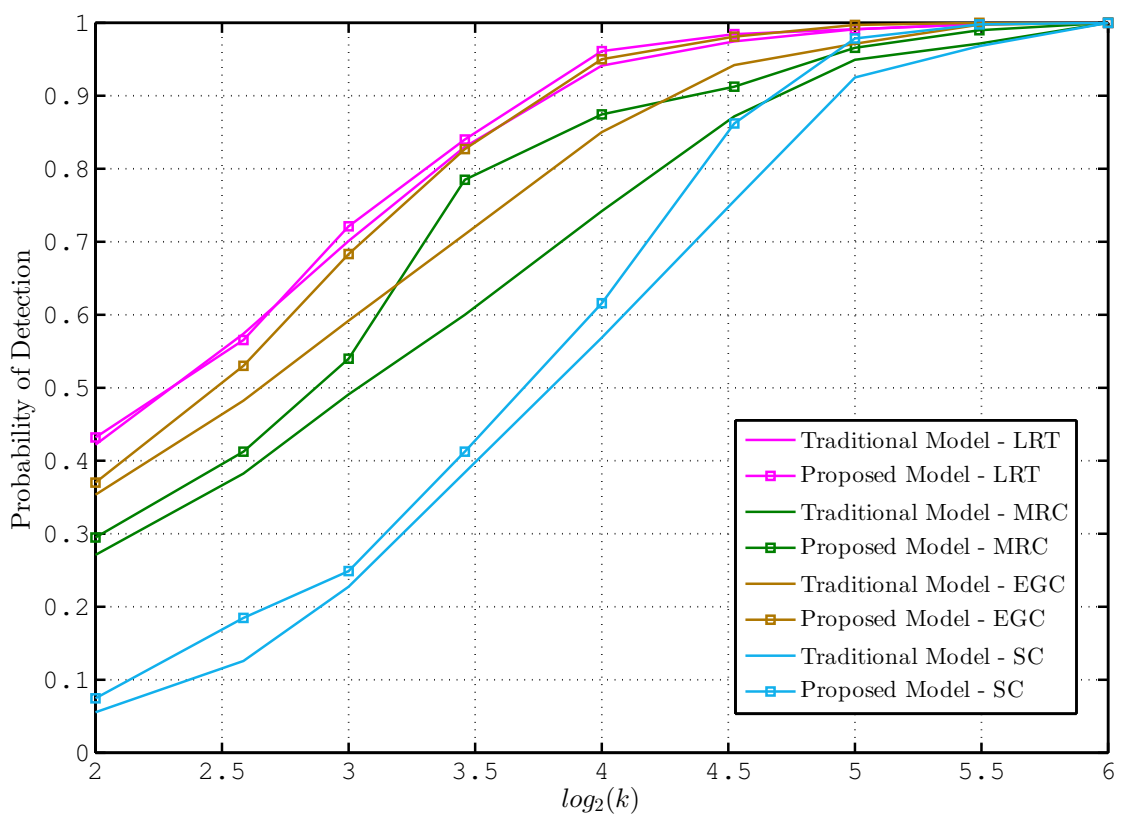

Figure 5. Probability of detection as a function of total number of sensors $\mathrm{K}, P_{d}=0.5, P_{f}=0.05$, and the average channel SNR is $5 \mathrm{~dB}$.

In the fourth simulation scenario, the effect of non-identical sensor performance indices is investigated. We assume that all sensors have the same probability of false alarm $\left(P_{f}=0.05\right)$ and a different probabilities of detection, where $\vec{P}_{d_{k}}=[0.1,0.2,0.3, \ldots, 0.8]$ and $K=8$. From Figure 6, the EGC fusion rule applied at the proposed model provides a relatively good performance when compared to SC and MRC and is similar to that of the LRT. Thus, the EGC fusion rule applied to the proposed WSN model could be a good alternative for the optimum LRT fusion rule applied at the FC.

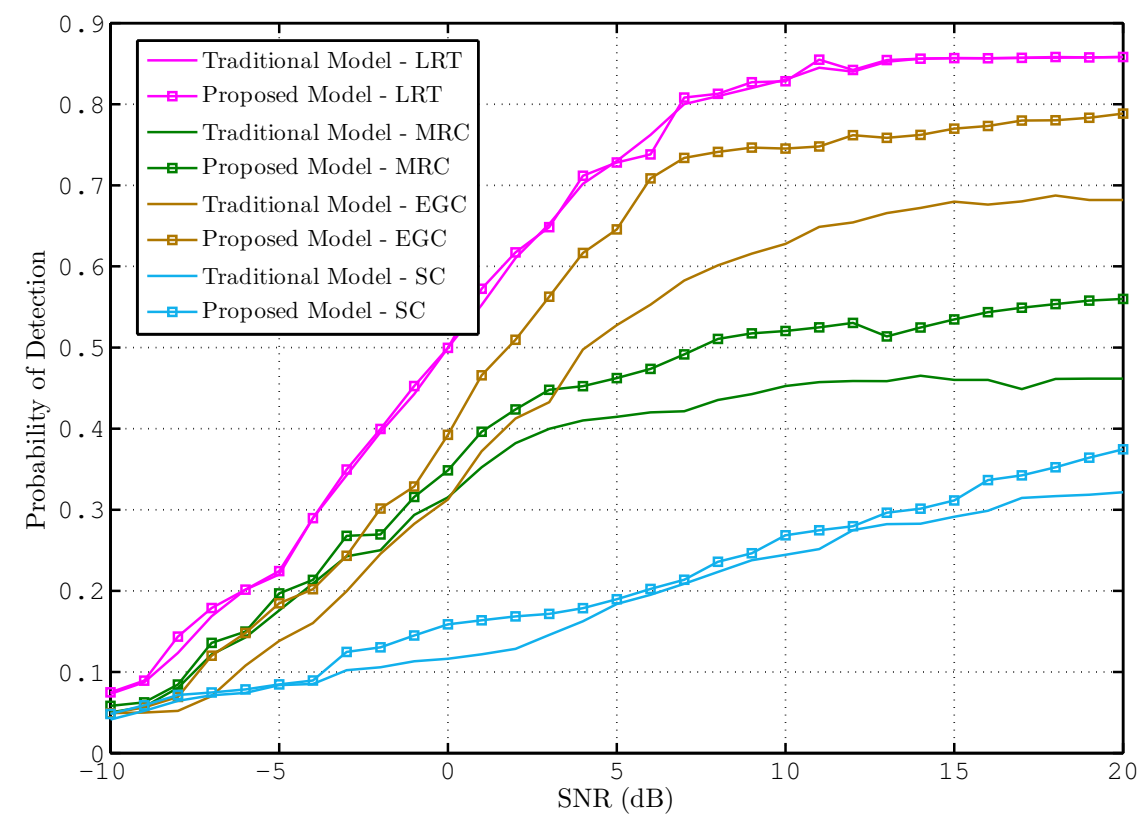

Figure 6. Probability of detection as a function of average channel SNR; sensors have different detection performance, total number of sensors $K=8$. 


\section{Conclusions}

In this paper, we propose an improved decision fusion model for WSNs over Rayleigh fading channels where the LRT is applied locally to each sensor. The application of the LRT at the sensors level improves the local decision and does not require either CSI or the transmission of local sensor performance indices from sensors to FC. This is essential for energy and bandwidth constrained WSNs. The improved local decision at each sensor enhanced the performance of different fusion rules when applied at the FC.

According to the comparative simulation study we that we carried out, the proposed WSN model outperforms the traditional one for all fusion rules considered and for a wide range of SNR. In addition, applying the EGC fusion rule at the FC of the proposed WSN model could be considered as a good alternative for the optimum LRT fusion rule as it provides a comparable performance to that of the LRT fusion rule and better performance than other fusion rules. Therefore, it is recommended to use the LRT at the sensor level and the EGC fusion rule at the FC.

Conflicts of Interest: The author declares no conflict of interest.

\section{References}

1. Akyildiz, I.F.; Su, W.; Sankarasubramaniam, Y.; Cayirci, E. Wireless sensor networks: A survey. Comput. Netw. 2002, 38, 393-422.

2. Khan, S.; Pathan, A.S.K.; Alrajeh, N.A. Wireless Sensor Networks: Current Status and Future Trends, 1st ed.; CRC Press, Inc.: Boca Raton, FL, USA, 2012.

3. Kumar, S.; Zhao, F.; Shepherd, D. Collaborative signal and information processing in microsensor networks. IEEE Signal Process. Mag. 2002, 19, 13-14.

4. Buratti, C.; Conti, A.; Dardari, D.; Verdone, R. An Overview on Wireless Sensor Networks Technology and Evolution. Sensors 2009, 9, 6869-6896.

5. Fahmy, H. Wireless Sensor Networks: Concepts, Applications, Experimentation and Analysis; Springer: Singapore, 2016.

6. Estrin, D.; Girod, L.; Pottie, G.; Srivastava, M. Instrumenting the world with wireless sensor networks. In Proceedings of the 2001 IEEE International Conference on Acoustics, Speech, and Signal Processing, Salt Lake City, UT, USA, 7-11 May 2001; pp. 2033-2036.

7. Kumar, S.; Shepherd, D. SensIT: Sensor information technology for the warfighter. In Proceedings of the 2001 International Conference on Information Fusion, Montreal, QC, Canada, 7-10 August 2001; Volume 1, pp. TuC1-TuC10.

8. Mainwaring, A.; Polastre, J.; Szewezyk, R.; Culler, D.; Anderson, J. Wirelesss sensor networks for habitat monitoring. In Proceedings of the 1st ACM Workshop on Wireless Sensor Networks and Applications, Atlanta, GA, USA, 28 September 2002; pp. 88-97.

9. Karl, H.; Willig, A. Protocols and Architectures for Wireless Sensor Networks; Wiley: Chichester, UK, 2005.

10. Chen, B.; Varshney, P.K. Bayesian sampling approach for decision fusion using hierarchical models. IEEE Trans. Signal Process. 2002, 50, 1809-1818.

11. Chair, Z.; Varshney, P.K. Optimal data fusion in multiple sensor detection systems. IEEE Trans. Aerosp. Electron. Syst. 1986, 22, 98-101.

12. Varshney, P.K. Distributed Detection and Data Fusion; Springer: New York, NY, USA, 1997.

13. Willett, P.K.; Swaszek, P.F.; Blum, R.S. The good, bad, and ugly: Distributed detection of a known signal in dependent Gaussian noise. IEEE Trans. Signal Process. 2000, 48, 3266-3279.

14. Chamberland, J.; Veeravalli, V.V. Decentralized detection in sensor networks. IEEE Trans. Signal Process. 2003, 51, 407-416.

15. Ciuonzo, D.; Romano, G.; Salvo, R.P. Decision Fusion in MIMO Wireless Sensor Networks with Channel State Information. In Proceedings of the IEEE Sensor Array and Multichannel Signal Processing Workshop (SAM '12), Hoboken, NJ, USA, 17-20 June 2012; pp. 17-20.

16. Ciuonzo, D.; Salvo, R.P.; Dey, S. Massive MIMO Channel-Aware Decision Fusion. IEEE Trans. Signal Process. 2015, 63, 604-619. 
17. Xiao, J.; Luo, Z. Universal decentralized detection in a bandwidth-constrained sensor network. IEEE Trans. Signal Process. 2005, 53, 2617-2624.

18. Ciuonzo, D.; Maio, A.; Salvo, R.P. A systematic framework for composite hypothesis testing of independent Bernoulli trials. IEEE Signal Process. Lett. 2015, 22, 1249-1253.

19. Liu, K.; Sayeed, A.M. Type-Based Decentralized Detection in Wireless Sensor Networks. IEEE Trans. Signal Process. 2007, 55, 1899-1910.

20. Li, W.; Dai, H. Distributed Detection in Wireless Sensor Networks Using A Multiple Access Channel. IEEE Trans. Signal Process. 2007, 55, 822-833.

21. Chen, B.; Tong, L.; Varshney, P.K. Channel-aware distributed detection in wireless sensor networks. IEEE Signal Process. Mag. 2006, 23, 16-26.

22. Chen, B.; Jiang, R.; Kasetkasem, T.; Varshney, P.K. Channel-aware decision fusion in wireless sensor networks. IEEE Trans. Signal Process. 2004, 52, 3454-3458.

23. Ciuonzo, D.; Salvo, R.P. Decision fusion with unknown sensor detection probability. IEEE Signal Process. Lett. 2014, 21, 208-212.

24. Li, F.; Evans, J.S.; Dey, S. Decision fusion over noncoherent fading multiaccess channels. IEEE Trans. Signal Process. 2011, 59, 4367-4380.

25. Ciuonzo, D.; Romano, G.; Salvo, R.P. Performance analysis and design of maximum ratio combining in channel-aware MIMO decision fusion. IEEE Trans. Wirel. Commun. 2013, 12, 4716-4728.

26. Salvo, R.P.; Ciuonzo, D.; Kansanen, K.; Ekman, T. Performance Analysis of Energy Detection for MIMO Decision Fusion in Wireless Sensor Networks Over Arbitrary Fading Channels. IEEE Trans. Wirel. Commun. 2016, 15, 7794-7806.

27. Wang, Y.; Xiong, M.; Yue, D.W.; He, R. Decision Fusion Rules over Rician Fading Channel for Wireless Sensor Networks. In Proceedings of the 2009 5th International Conference on Wireless Communications, Networking and Mobile Computing (WiCom '09), Beijing, China, 24-26 September 2009; pp. 1-4.

28. Niu, R.; Chen, B.; Varshney, P.K. Fusion of decisions transmitted over Rayleigh fading channels in wireless sensor networks. IEEE Trans. Signal Process. 2006, 54, 1018-1027.

29. Olver, F.W.J.; Lozier, D.W.; Boisvert, R.F.; Clark, C.W. NIST Handbook of Mathematical Functions; Cambridge University Press: Cambridge, UK; New York, NY, USA, 2010.

(C) 2017 by the authors; licensee MDPI, Basel, Switzerland. This article is an open access article distributed under the terms and conditions of the Creative Commons Attribution (CC BY) license (http:/ / creativecommons.org/licenses/by/4.0/). 